\title{
Surface-induced Dissociation of Molecular Ions in a Quadrupole Ion Trap Mass Spectrometer
}

\author{
S. A. Lammert and R. G. Cooks \\ Department of Chemistry, Purdue University, West Lafayette, Indiana, USA
}

A method is reported by which surface-induced dissociation is used to activate ions stored in a quadrupole ion trap mass spectrometer. The method employs a short $(<5 \mu s)$, fast-rising ( $<20$-ns rise time), high voltage direct current (dc) pulse, which is applied to the endcaps of a standard Paul-type quadrupole ion trap. This is in contrast to the application of an alternating current (ac) signal normally used to resonantly excite and dissociate ions in the trap. The effect of the dc pulse is to cause the ions rapidly to become unstable in the radial direction and subsequently to collide with the ring electrode. Sufficient internal energy is acquired in this collision to cause high energy fragmentations of relatively intractable molecular ions such as pyrene and benzene. The dissociations of limonene are used to demonstrate that high energy demand processes increase in relative importance in the dc pulse experiment compared with the usual resonance excitation method used to cause activation. The fragments are scanned out of the ion trap using the conventional mass-selective instability scan mode. Simulations of ion motion in the trap provide evidence that surface collisions occur at kinetic energies in the range of tens to several hundred clectronvolts. The experiments also demonstrate that production of fragment ions is sensitive to the phase of the main radiofrequency drive voltage at the point when the $\mathrm{dc}$ is initiated. (J Am Soc Mass Spectrom 1991, 2, 487-491)

I nteractions between polyatomic ion beams and surfaces [1-3] open reaction channels including charge [4-6] and atom [7] transfer and raise interesting questions about reaction dynamics $[8,9]$. These interactions are also of practical significance, especially in plasma etching and other materials processes encountered in the electronics industry [10].

Studies on the collisions of beams of ions with surfaces build on a large base of information on atomic and molecular collisions of gaseous ions [11-16]. One of the most important of these experiments is collision-activated dissociation (CAD) [17-20], a process often used in tandem mass spectrometry (MS/MS) to characterize molecular ions by causing their dissociation and recording the fragment ions generated. So important is the CAD experiment that four-sector instruments are used to study dissociation in kiloelectronvolt energy collisions [21, 22], but in spite of this, molecules of $>3000 u$ in molecular weight are seldom successfully fragmented. Because of these limitations there is interest in alternative means of dissociation, including photodissociation [23-25] and surface-induced dissociation (SID). The SID experiment has been done using quadrupole, sector, and time-offlight tandem mass spectrometers [26-32]. Recently, two groups have reported its implementation in the

Address reprint requests to R. G. Conks, Department of Chemistry, Purdue University, West Lafayette, IN 47907-3699. ion cyclotron resonance (ICR) mass spectrometer [33, 34]. It is therefore appropriate to attempt to develop a method for causing ion/surface collisions within a quadrupole ion trap $[35,36]$, particularly as rapid developments of the capabilities of this device have recently been made. These include a procedure for mass-selectively exciting and dissociating trapped ions, extension of the mass/charge range, and development of capabilities for ionization of nonvolatile compounds [37-40].

Although very useful in many ways, the MS/MS characteristics of the quadrupole ion trap have some limitations. Among these is the fact that the amount of internal energy which can be supplied to the trapped ion is relatively small. Estimates may be made of the distribution of internal energies supplied to selected molecular ions by CAD on the basis of the extent to which they undergo a sequence of consecutive dissociations [41]. In the ion trap, operated under normal conditions, distributions of internal energies with maximum values of $\sim 6 \mathrm{eV}$ have been measured [42] and access to higher internal energies is only possible by accepting large reductions in efficiency. Normal activation conditions are not adequate to cause significant fragmentation of such refractory ions as ionized pyrene [43], so the motivation to implement methods that transfer more internal energy is particularly strong. The experiment described here allow mass-selected ions to undergo surface collisions and 
the products of the collision to be collected and identified in an MS/MS experiment by using the ion trap.

\section{Experimental}

Experiments were performed by using a prototype ion trap mass spectrometer (ITMS) (Finnigan-MAT, San Jose, CA) [42] operated in the electron-impact mode. The ion trap is operated by using helium as the buffer gas at a nominal measured pressure of $1 \times 10^{-4}$ torr. Experiments were performed on four compounds: pyrene, limonene, $n$-butyl benzene, and benzene. Samples were introduced neat into the vacuum chamber of the ITMS by using either a heated solids probe (pyrene), or a Granville-Phillips leak valve (limonene, $n$-butylbenzene, and benzene). All compounds were obtained from commercial sources and used as supplied.

The molecular ion of interest was isolated in the ion trap by applying appropriate radiofrequency (rf) and direct current (dc) voltages to the ring electrode. Conventional $\mathrm{CAD}$ experiments employed Mathieu parameters $q_{z}$ of $0.2-0.4$, supplementary resonance amplitudes of up to $3 \mathrm{~V}$ (peak to peak), and irradiation times of $20 \mathrm{~ms}$. The new dc pulse experiments subjected the stored molecular ions to a rapid dc pulse applied to the endcaps of the ion trap analyzer. The pulses were of variable amplitude, typically $300-400 \mathrm{~V}$, applied for 1-4 $\mu \mathrm{s}$. They were generated by a home-built de pulser and controlled by a TTL signal derived from the ITMS control system. The high speed pulser itself consists of a custom-built circuit to provide a TTL control pulse which is phaselocked to the fundamental $\mathrm{rf}$ and controls the pulse width. The high voltage is provided by a Bertan model 1500-P variable high voltage supply (Hicksville, NY) which is externally controlled. The high voltage pulse stage uses a Directed Energy, Inc., model HV1000 pulser (Ft. Collins, Co) which takes the desired voltage from the Bertan supply and the TTL controlling pulse and generates the fast dc pulse which is then simultaneously applied to both endcaps via a $50-\Omega$ load splitter. The pulser is capable of delivering a $0-950 \mathrm{~V}$ pulse with a duration that is variable in the range 0.07-10 $\mu \mathrm{s}$. The phase-lock to the $1.1 \mathrm{MHz}$ fundamental if voltage (applied to the ring electrode) is implemented in such a way as to allow the pulse to be initiated at any point throughout the rf cycle.

\section{Results and Discussion}

Polynuclear hydrocarbons have been examined by a variety of ionization and activation methods [43]. Under conditions of low collision energy, fragmentation is limited to loss of $\mathrm{H} \cdot, \mathrm{H}_{2}, \mathrm{C}_{2}$-containing fragments and to a few other ions in the high mass region. The ion trap product (daughter) spectrum of the pyrene molecular ion, $m / z 202$, recorded under normal trap- ping and irradiation conditions, gave results consistent with this, being completely dissociated but displaying only $\mathrm{H} \cdot$ and $\mathrm{H}_{2}$ loss ions in $45 \%$ and $100 \%$ relative abundance, respectively. On the other hand, when activation is caused by application of the $\mathrm{dc}$ pulse instead of the normal supplementary alternating current (ac) resonance voltage, the spectrum shown in Figure 1 was recorded. In this experiment the dc pulse was applied to the endcaps at $325 \mathrm{~V}$ for $3.65 \mu \mathrm{s}$, while the main rf amplitude was set to correspond to a Mathieu parameter $q_{2}$ of 0.43 . The ions which appear at $m / z 122,150,151,174$, and 175 were not present in the conventional product spectrum. The fragment ions at masses less than $m / z 190$ are characteristic fragments of highly excited pyrene molecular ions; for example, the fragment $m / z 150$ is estimated to have an appearance energy of $11 \mathrm{eV}$, while $\mathrm{m} / \mathrm{z} 122$ requires $17 \mathrm{eV}$ [44]. The relative abundances of these ions under the conditions of Figure 1 are not high, the most abundant being only $3 \%$ of the most abundant fragment $(m / z 200)$ and just $0.2 \%$ of the $m / z 202$ ion. Moreover, the $q_{z}$ value used corresponds to $\mathrm{m} / \mathrm{z} 95$, being the lowest fragment in mass stored; so there is likely to be significant mass discrimination in recording the product spectrum.

To obtain more insight into ion motion in the trap under the conditions used here, ion trajectories were simulated by using a program developed in our laboratory [45]. The program has been found to give good results in comparison with experiments that measure the kinetic energies with which ions are ejected from the trap [46]. Figures 2 and 3 display both the radial and axial positions and velocities for the pyrene molecular ion in the dc pulse experiment described above. The radial simulation shows that $-2 \mu \mathrm{s}$ after the initiation of the dc pulse the pyrene molecular ion strikes the ring electrode. The collision energies in this simulation depend heavily on the phase and position of the ion when the pulse is applied, but the typical data shown demonstrate that over $100 \mathrm{eV}$ of

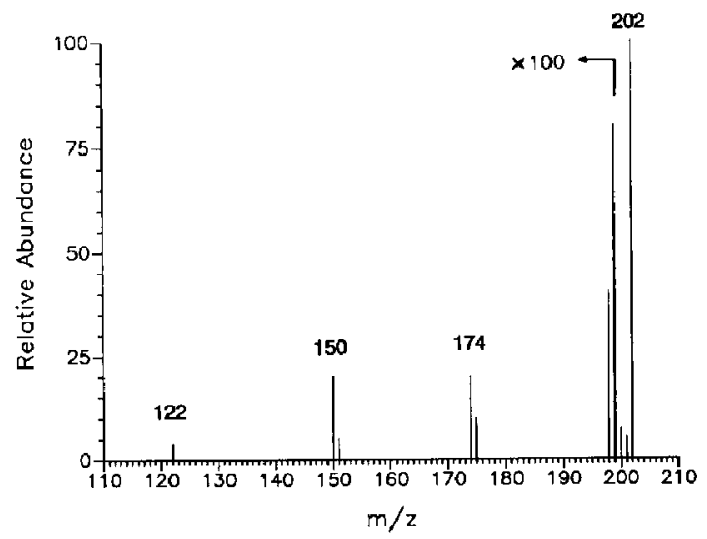

Figure 1. Product spectrum of pyrene molecular ion recorded after applying a 325-V dc pulse for $3.65 \mu \mathrm{s}$ at $q_{z}=0.43$. 


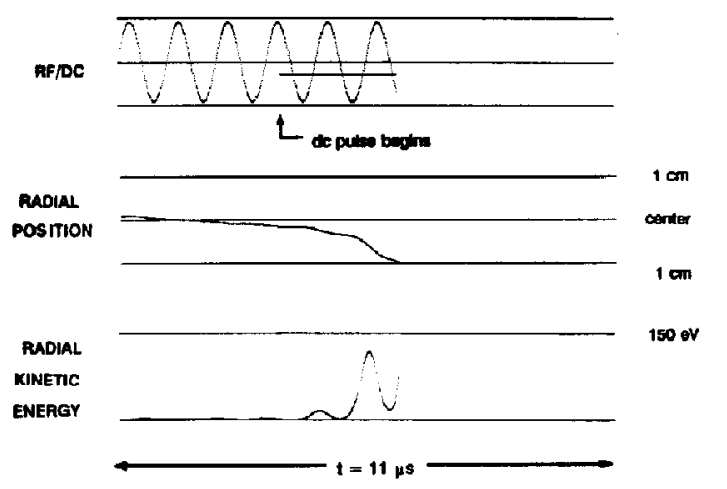

Figure 2. Simulation of motion of pyrene molecular ion in the radial direction. Individual parts of the figure show the applied potentials, the radial position and radial component of the ion kinctic encrgy. Conditions match those used for the experiment in Figure 1. The simulation ends when the ion strikes the ring electrode.

radial translational kinetic energy are available. The simulation does not specifically include the effects of collisions with helium, but at a pressure of $10^{-3}$ torr the collision frequency is on the order of one collision in $10 \mathrm{rf}$ cycles. These same simulations show that the ions strike the surface within $<10 \mathrm{rf}$ cycles after the pulse initiation, so that typically zero or one collision with helium occurs during the activation time. Simulation of motion in the axial dircction (Figure 3) shows that the pyrene molecular ions remain trapped in this direction and that the axial kinetic energies are smaller. Substantially similar results are obtained when other experimental conditions are simulated. The axial component of the kinetic energy increases with the value of $q_{z}$ as expected from the Dehmelt potential well model [47]. The simulations clearly support the suggestion that dissociation is due to collisions with the walls, that is, to SID. However, gaseous CAD processes may also contribute to the observed fragmentation.

Another system that has been extensively studied by various activation techniques is the molecular ion of limonene $[48,49]$. These studies, which have included low energy gaseous and surface collisions, kilovolt energy $C A D$, angle resolved mass spectrometry, and photon- and electron-stimulated dissociation, have allowed the energy requirements for various fragment ions to be ranked. Among the ions whose formation requires higher internal energies are $\mathrm{m} / \mathrm{z}$ 77 and 91, whereas other ions with lower energy requirements include $m / z 94$ and 79 . The results (Figure 4) of activation in the ion trap by the methods of $\mathrm{dc}$ and conventional ac excitation are similar except that the two high energy ions, $m / z 77$ and 91 , virtually absent under normal activation conditions, make a substantial contribution to the spectrum recorded under the conditions of dc activation.

The most direct evidence that SID is contributing to the product MS/MS spectra when the dc pulse is

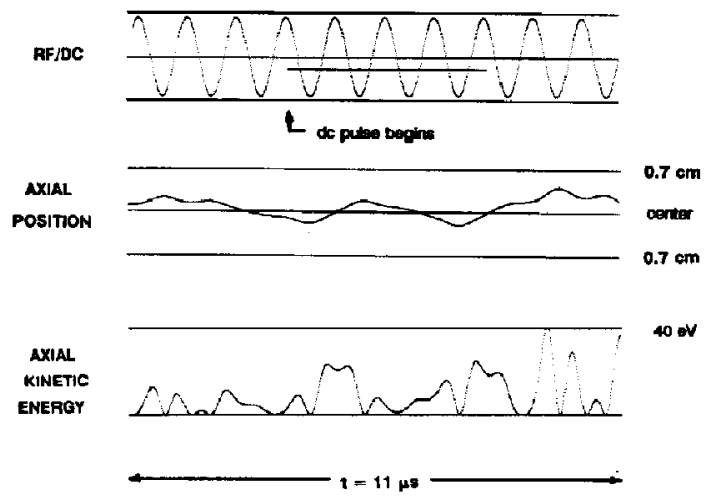

Figure 3. Simulation of motion of pyrene molecular ion in the axial direction. Individual parts of the figure show the applied potentials, the axial position, and the axial component of the ion kinetic energy. Conditions match those used for the experiment in Figure 1.

applied is found in data taken for the benzene molecular ion. Fragmentation occurs to give $m / z 52,51$, and 50, among other ions. These data are similar to results obtained using SID on a conventional quadrupole instrument [50]. When a delay is introduced between the maximum of the main $\mathrm{rf}$ cycle and the onset of application of the dc pulse, the abundances of these fragment ions vary with the magni-

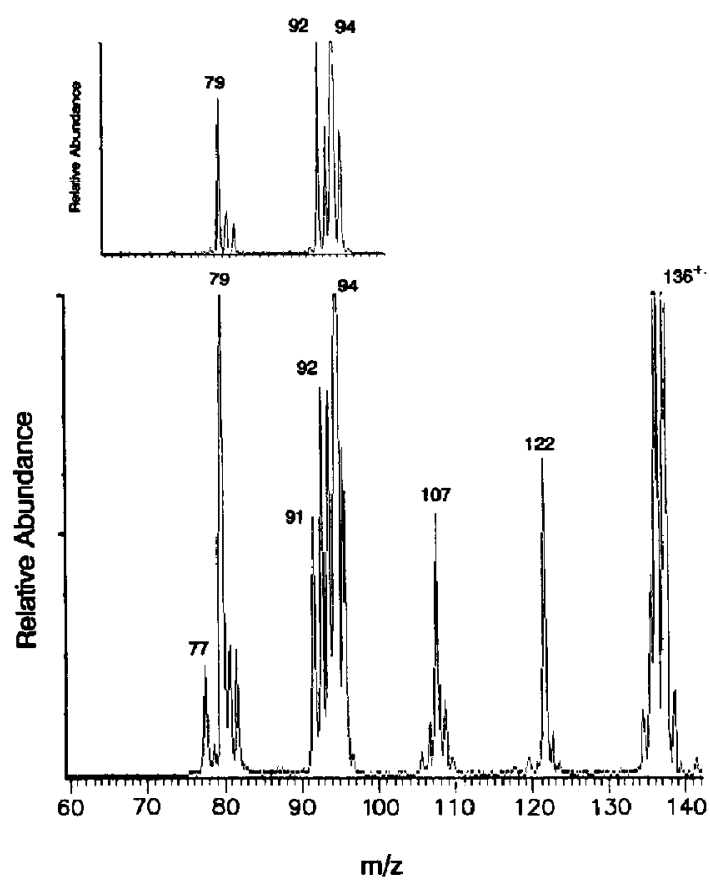

Figure 4. Product spectra of the limonene molecular ion after application of a dc pulse ( $\left.305 \mathrm{~V}, 2.3 \mu \mathrm{s}, q_{z}=0.5\right)$. Inset shows a typical CAD spectrum produced by the conventional method of resonance excitation (ac $=700 \mathrm{mV}$ at $91545 \mathrm{~Hz}$ for $3 \mathrm{~ms}, q_{z}=$ $0.23)$ for the mass range 65 to 100 . 
tude of the delay in the fashion shown in Figure 5. The ion abundance maxima occur at intervals which correspond to one full rf cycle. Clearly, the interaction between the fields created by the $\mathrm{dc}$ and the main rf voltages has a strong effect on ion production. This result is consistent with the SID process because the main rf field will alternately assist and resist acceleration of the parent ions to the radial electrode by the axial de potential.

\section{Conclusions}

Although previous studies have demonstrated that SID contributes to the fragmentation observed in ion injection experiments $[51,52]$ and despite suspicions that even conventional resonance excitation in MS/MS involves surface collisions [Glish, G. L., personal communication, January 1991], this is the first study in which SID is demonstrated for trapped ions. Dissociation is deliberately produced by accelerating the trapped ions to the ring electrode without using resonance excitation. Collision energies are estimated from the simulations to be in the hundreds of electron volts and the process results in the observation of fragments with high threshold energies. Like the recent SID results obtaincd in ICR instruments $[33,34]$, the experiment is still at a rudimentary stage, especially in terms of efficiency of fragmentation. It is also apparent from the results obtained on limonene and $n$-butyl benzene (not discussed above) that a relatively wide range of internal energies is deposited in the activated parent ion. In part this may be due to contributions from CAD processes. However, the simulations suggest an additional interpretation, namely that collision energy and angle vary greatly with the initial (pre-dc pulse) conditions of the stored ion and hence a range

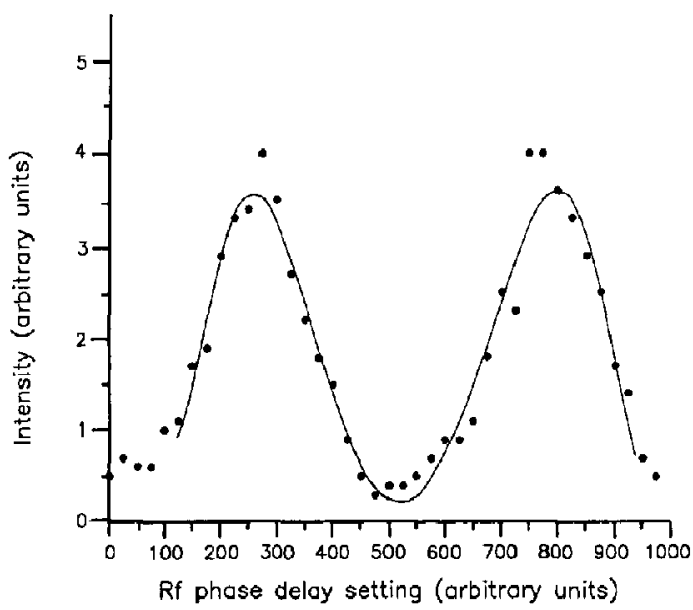

Figure 5. Abundance of the $m / z 52$ fragment ion generated from the benzene molecular ion as a function of the rf phase delay between an arbitrary point in the rf phase and application of a dc pulse $(350 \mathrm{~V}, 1.5 \mu \mathrm{s})$. of internal energy depositions can be expected. This range will be increased if, as is probable, collection of the ions from the surface into stable trajectories for subsequent mass analysis is subject to discrimination based on the direction or velocity of ejection.

\section{Acknowledgments}

This work was supported by the National Science Foundation, CHE 87-21768. The authors thank Hans-Peter Reiser for assistance with the simulations.

\section{References}

1. Kaminsky, M. Atomic and lonic Impact Phenomena on Metal Surfaces; Academic: New York, 1965.

2. Kasi, S. R.; Kang, H.; Sass, C. S.; Rabalais, J. W. Surf. Sci. Rep. 1989, 10, 1.

3. Cooks, R. G.; Ast, T.; Mabud, M. A. Int. I. Mass Spectrom. Ion Processes 1990, 100, 209.

4. van Zoest, J. M.; van der Meij, C. E.; Fluit, J. M. Nucl. Instrum. Methods 1986, B13, 587.

5. Kang, H.; Schuler, T. R.; Rabalais, J. W. Chem. Phys. Rev. 1986, 128,348 .

6. Vincenti, M.; Iloming, S. R,; Cooks, R. G. Org. Muss Spectrom. 1988, 23, 585.

7. Ast, T.; Mabud, M. A.; Cooks, R. G. Int. I. Mass Spectrom. Ion Processes 1988, 82, 131.

8. Snowdon, K. Nucl. Instrum. Methods 1988, B33, 365.

9. Heiland, W.; Beitat, W.; Taglauer, E. Phys. Rev. 1979, 19, 1677.

10. Coburn, J. W.; Winters, H. F. J. Appl. Phys. 1979, 50, 3189.

11. Hasted, J. B. Physics of Atomic Collisions, 2nd ed.: Elsevier: New York, 1972.

12. Cooks, R. G., Ed. Collision Spectroscopy; Plenum; New York, 1978.

13. Graul, S. T.; Squires, R. R. Mass Spectrom. Rev. 1987, 7, 263.

14. Russell, D. H. Gas Phase Inorganic Chemistry; Plenum: New York, 1989.

15. Buchanan, M. V. Fourier Transform Mass Spectrometry; ACS Symposium Series 359; American Chemical Society: Washington, DC, 1987.

16. Ast, T. Adv. Mass Spectrom. 1986, 10, 471.

17. Bordas-Nagy, J.; Jennings, K. R. Int. J. Mass Spectrom. Ion Processes 1990, 100, 105.

18. McLafferty, F. W., Ed. Tandem Mass Spectrometry; Wiley: New York, 1983.

19. Levsen, K.; Schwarz, H. Mass Spectrom. Rev. 1983, 2, 77.

20. Busch, K. L.; Glish, G. L.; McLuckey, S. A. Mass Spectrometry/Mass Spectrometry, Techniques and Applications of Tandem Mass Spectrometry; VCH: New York, 1988.

21. Desiderio, D., Ed. Mass Spectrometry of Peptides; CRC: Boca Raton, FL, 1991.

22. Biemann, K.; Martin, S. A. Mass Spectrom. Rev. 1987, 6, 1.

23. Grotemayer, J.; Schlag, E. W. Acc. Chem. Res. 1989, 22, 399.

24. Lubman, D. M., Ed. Lasers and Mass Spectrometry; Oxford: New York, 1990.

25. Dunbar, R. C. In Gas Phase Ion Chemistry, Vol. 3; Bowers, M. T., Ed.; Academic: Orlando, FL, 1984 .

26. Mabud, M. A.; DeKrey, M. J.; Cooks, R. G. Int. J. Mass Spectrom. Ion Processes 1985, 67, 285.

27. Bier, M. E.; Amy, J. W.; Cooks, R. G.; Syka, J. E. P.; Ceja, P.; Stafford, G. Int. J. Mass Spectrom. Ion Processes 1987, 77, 31.

28. Schey, K.; Cooks, R. G.; Grix, R.; Wollnik, H. Int. J. Mass Spectrom. Ion Processes 1987, 77, 49. 
29. Bier, M. E.; Schwarz, J. C.; Schey, K. L.; Cooks, R. G. Int. J. Mass Spectrom. Ion Processes 1990, 103, 1.

30. Aberth, W. Anal. Chem. 1990, 13, 609.

31. LeMeillour, C.; Cole, R.; Clairet, F.; Fournier, F.; Tabet, J. C.; Blasco, T.; Beaugrand, C.; Devant, G. Adv. Mass Spectrom. 1989, 11, 236.

32. Wysocki, V. H.; Ding, J. M.; Jones, J. L.; Callahan, J. H.; King, F. L.; J. Am. Soc. Mass Spectrom. (in press).

33. Ijames, C. F.; Wilkens, C. L. Anal. Chem. 1990, 13, 1295.

34. Williams, E. R.; Henry, K. D.; McLafferty, F. W.; Shabanowitz, J.; Hunt, D. F. I. Am. Soc. Mass Spectrom. 1990, 1, 413.

35. Paul, W.; Steinwedel, H. U.S. Patent 2939952, June 7, 1960.

36. March, R. E.; Hughes, R. J. Quadrupole Storage Mass Spectrometry; Wiley: New York, 1989.

37. Cooks, R. G.; Kaiser, R. E. Jr. Acc. Chem. Res. 1990, 23, 213.

38. Glish, G. L.; Mcluckey, S. A. Anal. Chem. 1990, 62, 1284.

39. Johnson, J. V.; Yost, R. A.; Kelley, P. E.; Bradford, D. C. Anal. Chem. 1990, 62, 2162.

40. Todd, J. F. J. Mass Spectrom. Rev. 1991, 10, 3.

41. Wysocki, V. H.; Kenttämaa, H. I.; Cooks, R. G. Int. J. Mass Spectrom. lon Processes 1987, 75, 181.
42. Louris, J. N.; Cooks, R. G.; Syka, J. E. P.; Kelley, P. E.; Stafford, G. C.; Todd, J. F. J. Anal. Chem. 1987, 59, 1677.

43. Pachuta, S. J.; Kenttämaa, H. I.; Sack, T. M.; Cerny, R. L.; Tomer, K. B.; Gross, M. L.; Pachuta, R. R.; Cooks, R. G. J. Am. Chem. Soc. 1988, 110, 657.

44. Nourse, B. N.; Cox, K. A.; Cooks, R. G. Submitted to J. Am. Chem. Sac.

45. Reiser, H.-P.; Julian, R. K.; Cooks, R. G. Manuscript to be submitted.

46. Reiser, H.-P.; Kaiser, R. E.; Savickas, P. J.; Cooks, R. G. Int. J. Mass Spectrom. Ion Processes, 1991, 106, 237.

47. Major, F. G.; Dehmelt, N. G. Phys, Rev. 1968, 190, 91.

48. Vincenti, M.; Horning, S. R.; Cooks, R. G. Org. Mass Spectrom. 1988, 23, 585.

49. Horning, S. R,; Wood, J. M.; Gord, J. R.; Freiser, B. S.; Cooks, R. G. Int. I. Mass Spectrom. Ion Processes 1990. 101, 219.

50. Hayward, M. J.; Mabud, M. A.; Cooks, R. G. I. Am. Chem. Soc. 1988, 110, 1343.

51. Morand, K. L.; Horning, S. R.; Cooks, R. G. Int. J. Mass Spectrom. Ion Processes, 1991, 105, 13.

52. Schwartz, J. C.; Kaiser, R. E. Jr.; Cooks, R. G.; Savickas, P. J. Int. J. Mass Spectrom. Ion Processes 1990, 98, 209. 